\title{
Democratizing IT: A Cloud Computing Framework
}

\author{
Jitendra Singh* $^{*}$ \\ IT Department, Asia Pacific Institute of management, Plot No. 3-4, Institutional Area Jasola, New Delhi- \\ 110025, India
}

\begin{abstract}
Information technology (IT) is indispensable in the present day business scenario and equally sought by established as well as the new enterprises. Consequently, need is to democratize the IT resources in order to offer enough opportunities to everyone to grow. A new emerging paradigm termed as cloud computing is attempting to democratize the IT procurement and usage. This work has proposed a framework that maps the democratic values offered with the help of cloud computing. Various key features are mapped that are enablers in the proliferation of the democratic values including equal opportunity, share-ability and minimum upfront cost. Finally, this article also highlights the key initiatives by the various governments in order to promote democratic values through the cloud computing.
\end{abstract}

Index Terms: Empowering subscriber, democratizing cloud computing, governance.

(C) 2016 Published by MECS Publisher. Selection and/or peer review under responsibility of the Research Association of Modern Education and Computer Science.

\section{Introduction}

Democracy empowers every citizen to elect the government that in turn manages the thing for them. Similarly, a new paradigm termed as Cloud computing also empowers its subscribers by offering powerful, huge resources. In cloud computing, resources capabilities are measured in terms of processing power, bandwidth, and memory (Risch \& Altmann, 2009). Usage of these cloud resources are charged on utility based model (Yeo, Buyya, Assuncao et al, 2007; Risch, Altmann, Guo,\& Fleming et al, 2009). In Cloud paradigm, resources are managed and maintained by the cloud provider. Consequently, data owners are free from resource management and can concentrate on business issues, instead of IT (Rappa, 2004; Buyya, Yeo,Venugopal et al, 2009).

Cloud computing has been defined by the number of experts and organizations active in this area including Armbrust et al. (2009), Foster, Zhao, Raicu \& Lu( 2008), Cafaro \& Aloisio(2011), Gartner etc. The one widely accepted is suggested by the National Institute of standard and technology (NIST) in its draft 800-145(Mell \&

* Corresponding author.

E-mail address: jitendra.singh0705@gmail.com 
Grant, 2010). NIST definition is also accepted by the cloud security alliance (CSA, 2009). NIST definition categorizes the cloud deployment into three models i) private cloud ii) public cloud iii) community cloud and iv) Hybrid cloud. In private cloud, resources are under the control of the users, whereas in public cloud, resources are under the control of cloud service provider (CSP), Hybrid cloud uses the combination of both the private and public cloud. NIST definition also categorizes the service models into i) Infrastructure as a Service (IaaS) ii) Platform as a Service (PaaS) iii) Software as a Service (SaaS). In the IaaS model, resources, for instance processing capabilities, memory and bandwidth are offered as services. Development environment is offered by the PaaS; whereas in SaaS model, software is offered as a Service. All these services can be offered by any type of cloud deployment model (private, public etc.).

Cloud computing is not altogether a new paradigm; instead it is evolved from some of the existing technologies like Grid, web 2.0, Service oriented architecture (SOA), etc. It makes the wide usage of virtualization in order to achieve the optimum utilization of resources. Cloud data center is an example that is using the aforementioned technologies (Singh, 2014-b). Cloud data centers are responsible for offering the computational resources needed as services under different cloud deployment models. Data center are spread across the world and are in the direct control of the cloud provider (Singh \& Kumar, 2013,a). Cloud data center are democratic in real sense and offers a lot of flexibility in resource subscription. Resources subscribed can be increased or decreased by the subscribers of their own with minimal or no intervention from the owner.

Cloud computing also offers tremendous scalability and agility (Lin, Fu, Zhu, \& Dasmalchi, 2009). Agility means resources can be obtained in hours instead of week and month time that is generally needed in the legacy system (Singh \& Kumar, 2014). As a result, additional resources needed for short duration can be demanded within short notice. Resources demanded can be released by the user once the requirement/spike is over (Singh \& Kumar, 2011). Expectation of minimum upfront cost to start the IT services can further attract the entrepreneurs towards this field.

To promote the business there is a need to offer equal opportunities to every aspirant. It is of profound importance and much needed in democratic setup. Rest of this manuscript is organized as: Section 2 discusses the major cloud providers active in the area of cloud computing and their prominent services. Section 3 discusses the various services subscribed and pattern of their utilization. In addition, it also discusses the major predictions by the prominent research and analysis organizations. Section 4, discusses the way cloud computing is active in promoting the democratic values and bridging the cross country barriers. Section under consideration has also highlighted the various cloud offerings facilitate in proliferating the democratic values. Finally, it discusses the initiative taken by various governments in order to serve and empower their citizens by leveraging the cloud computing.

\section{Major Cloud Providers}

Cloud computing has huge growth potential that is evident from many of the recent research reports published by the major research and analytical groups that include Gartner, IDC, Aberdeen, etc. In a recent survey of IDC, cloud computing has been identified as one of the major technologies set to dominate the IT arena in the upcoming decade. Realizing this huge demand and business potential, a number of vendors have emerged and are offering cloud services.

Amazon, Microsoft and Google are the prominent cloud providers with major user share (Curtis, 2014). With the passage of time, cloud providers are specializing in their area of offerings. For instance, Amazon is increasingly appreciated for its infrastructure as a service via public cloud. Similarly, goGrid is specialized in its infrastructure as a service offering through its public cloud. Microsoft, another major cloud giant is offering both private and public cloud to the users. Salesforce has emerged as SaaS service provider through the public cloud model. Major cloud providers and their major services have been enumerated in the upcoming subsection: 


\subsection{Amazon}

Amazon is the major cloud provider and has dominance in the IaaS. Under its IaaS offering, it offers compute power such as processor, RAM, bandwidth, etc. for subscription. It also offers storage as a service under this category with the nomenclature Simple storage services (S3) in which users can store data at Amazon (Curtis, 2014). Amazon S3 is widely appreciated by the subscribers lacking the storage capabilities at their own premises. Amazon is also offering monitoring tool 'Cloud watch' a proprietary tool to monitor the cloud health and usage at any specific point in time.

In addition, Amazon is also offering various application services in order to meet the requirement of variety of users. Offered services serve the need of wide variety users, services offers include, Queue service, Notification services and workflow services, etc.

\subsection{Google}

Web giant Google is the other major player in cloud computing domain and registers it presence in all types of cloud deployment model and service offerings. A Google doc is the popular SaaS based services offered by the Google to the web user. Users can use the word processor, presentation and spreadsheet offered by the Google from its Google doc. To share the pictures and video, Google offers Picasa that is widely appreciated by the users under this segment. Recently, to facilitate users in conducting online meeting and to have live session, it also offers hang out, a considerable unique feature among its rivals. Users leveraging the Google chrome can use hang out without including any additional software.

Table 1. Deployment model and Their Services

\begin{tabular}{|l|l|l|}
\hline S.NO & Deployment model & Offerings \\
\hline 1 & IaaS & Google compute services \\
\hline 2 & PaaS & Google apps engine \\
\hline 3 & SaaS & Picasa, Google docs, blog, hang-out etc. \\
\hline
\end{tabular}

\subsection{Microsoft}

Microsoft is another major player under this category with windows azure as its popular offering. Windows azure is an integral platform that offers infrastructure as a Services, Platform as a Services and Software as a Service. Windows azure allows the users to select the cloud model that is more appropriate for them.

In addition, Microsoft has also made its presence felt in the private cloud domain. Organizations that are hesitant to opt public model of cloud, but still intend to use cloud computing, private cloud is much sought by them. Private cloud model facilitates in maintaining the privacy and realizing the benefit of cloud computing. Microsoft is also offering its popular office through the cloud. This software can be utilized by the user on utility model. To cater the mobile phone users, it is also offering office 365 , which is an office suite application.

\subsection{GoGrid}

The other major cloud provider offers only Infrastructure as a Service. It is considered as highly reliable relative to other providers in this segment and guarantees the uptime of 99.999. Gogrid also claims the explicit 
support to big data (www.gogrid.com). To provide the better performance it also supports Solid state drive (SSD) storage. Usage charges for gogrid cloud are enumerated in the following table:

Table 2. Gogrid Servers and Pricing

\begin{tabular}{|l|l|l|l|l|l|l|}
\hline $\begin{array}{l}\text { SSD Cloud } \\
\text { Servers }\end{array}$ & RAM & Cores & $\begin{array}{l}\text { Storage } \\
(\mathrm{GB})\end{array}$ & Hourly & $\begin{array}{l}\text { Monthly } \\
(\text { Save 25\% })\end{array}$ & $\begin{array}{l}\text { Annual } \\
(\text { Save 50\% })\end{array}$ \\
\hline Small & $2 \mathrm{~GB}$ & 2 & 80 & $\$ 0.15$ & $\$ 82.13$ & $\$ 657.00$ \\
\hline Medium & $4 \mathrm{~GB}$ & 4 & 160 & $\$ 0.30$ & $\$ 164.25$ & $\$ 1,314.00$ \\
\hline Large & $8 \mathrm{~GB}$ & 8 & 320 & $\$ 0.60$ & $\$ 328.50$ & $\$ 2,628.00$ \\
\hline X-Large & $16 \mathrm{~GB}$ & 16 & 640 & $\$ 1.20$ & $\$ 657.00$ & $\$ 5,256.00$ \\
\hline 2X-Large & $32 \mathrm{~GB}$ & 28 & 1,280 & $\$ 2.04$ & $\$ 1,116.90$ & $\$ 8,935.20$ \\
\hline 4X-Large & $64 \mathrm{~GB}$ & 40 & 2,199 & $\$ 2.99$ & $\$ 1,637.03$ & $\$ 13,096.20$ \\
\hline
\end{tabular}

\subsection{Other significant cloud providers}

In addition to the aforementioned cloud providers, many others provider are also offering their services and it would be worth mentioning about them. These Cloud providers are offering their services in the specific area, such as ZOHO that is functioning in the SaaS domain. Zoho is offering comprehensive SaaS services ranging from collaboration, invoice, ERP to database management. Similarly, Salesforce.com is also emerging as leader in the SaaS area of cloud computing. Many research organizations that include IDC, Aberdeen, Gartner, etc. have predicted huge growth for the Salesforce. Even at the present time, it is an established leader in the present day SaaS providers. Similarly, IT leaders such as IBM, HP, Oracle, etc. are also offering their services from their cloud and enjoying remarkable customer's base.

\section{Utilization of Cloud Resources and Predictions}

Usage of cloud computing is not uniform for all types of services instead varies significantly.

Subscription from small to large industry of the same type also varies. Correspondingly, the upcoming section highlights the cloud usage based on the services and industry size.

\subsection{Usage of cloud based on the services}

Enterprises are subscribing for the cloud services to meet their specific needs. Based on the services required it can be subscribed from private, public, hybrid or community cloud. However, public model of cloud is considered to be a true cloud model. Because, in this model of computing, resources are offered on utility model and the entire cost of maintenance is borne by the cloud provider. In public cloud, extra resources required are available in hours instead of weeks and month time needed in case of legacy system (Li, Yang, Kandula \& Zhang, 2010). This model offers the tremendous elasticity and gives the impression of infinite resources, although there is nothing like infinite exists in cloud data center. As a result, public cloud deployment model is particularly appealing to the cloud users intending to get rid of resource procurement and maintenance.

Within the public cloud, SaaS based offering has significant subscription from the users, that is followed by cloud storage and IaaS respectively. Public cloud model is highly subscribed by the small and medium-sized subscribers and same has been illustrated in the figure 1.

Large organizations are subscribing for the SaaS based application as well as hybrid cloud, whereas majority of them prefer to use in-house facilities for the other services due to security and regulatory compliances, refer figure 2 . 


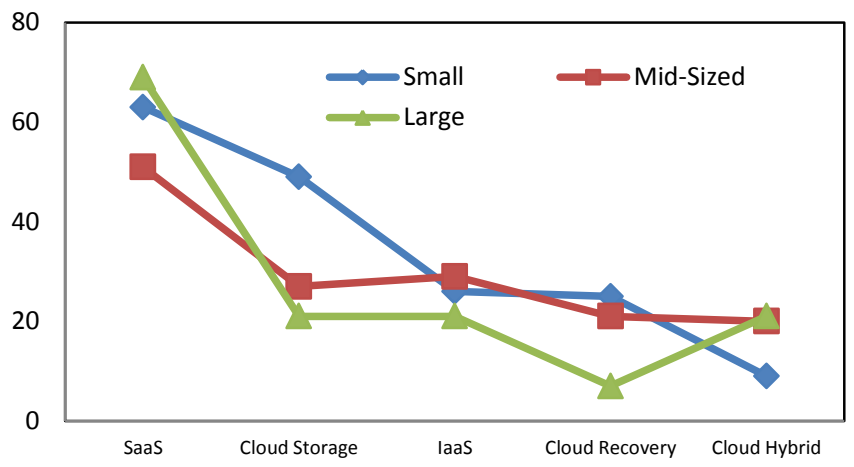

Fig.1. Cloud Service Utilization Based on Industry

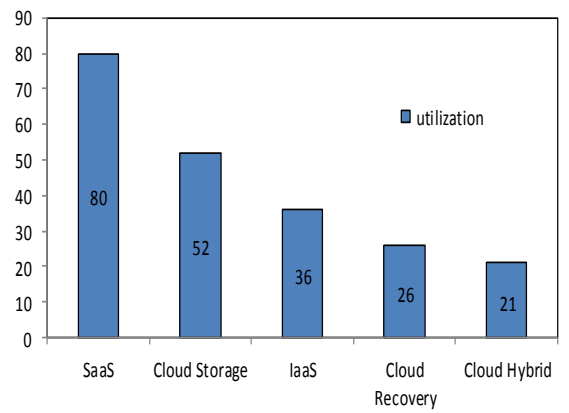

Fig.2. Services Share in Cloud Utilization

\subsection{Usage of cloud based on the industry size}

Usage of cloud computing is not uniform for the same type of enterprises instead varies based on the size of the industry i.e. small, medium and large, etc. For instance, SaaS based services such as build in applications are widely subscribed by the large enterprises relative to the medium and small enterprises. The key reason is the less risk involved in the usage of SaaS for the large organization, whereas, storage services and infrastructure services are widely subscribed by the small and medium enterprises in order to get relieved from the resource maintenance and to avoid the upfront cost. Same has been illustrated in the figure 2 .

\subsection{Cloud computing benefits and future prediction}

Cloud computing is set to revolutionized the way IT is owned and used. Considering the growth potential, IDC has placed it in the list of $3^{\text {rd }}$ platform. According to the IDC, $3^{\text {rd }}$ platform is set to dominate the technological arena in the upcoming decade. Third platform ( $3^{\text {rd }}$ Platform) encompasses of mobile devices, cloud computing, social technologies, and Bigdata.

According to the IDC, IT growth will be primarily driven by the $3^{\text {rd }}$ Platform that also includes the cloud computing (Gens, 2013). Similarly, by $202040 \%$ of industry revenue and $98 \%$ of its growth will be driven by 3rd Platform (Gens, 2013). Same report also predicted the spending by the developing countries would grow by $8.8 \%$ to exceed $\$ 730$ billion. According to the IDC report, price, risk and availability are defined as key control variables for the public cloud (pucciarelli, 2011). 
In the $3^{\text {rd }}$ annual future of cloud computing survey, North bridge venture partners and GigaOM research has projected the huge growth in cloud paradigm. According to the survey, $75 \%$ of those surveyed have already adopted the cloud computing in one form or the other, which is up from 67 percent from last year. An increase of $126.5 \%$ is to be witnessed in 2014 since 2011 in the cloud arena. Various other significant predictions include:

- 76 percent participants expect hybrid cloud would emerge to be the widely adopted cloud, surpassing private and public cloud.

- IaaS share has reached to $45 \%$ relative to $35 \%$ usage in the previous year.

- File sharing, business productivity, CRM, Collaboration, etc. are among the 16 applications that are widely subscribed by more than half of the surveyed company.

- Security risk is reduced from 55\% to only $46 \%$ in 2013, although still it remains the major inhibitor of cloud adoption.

IDC in its survey titling "Cloud Computing's Role in Job Creation", predicted that the majority of the job in the IT sector will be generated by the cloud computing. According to IDC 13.8 million, total cumulative jobs will be generated by the cloud computing by the year 2015 .

\section{Cloud Computing And Democracy}

Cloud computing is gaining ground both in the private and public sectors. Cloud paradigm has significant role in strengthening the democratic values in IT usage. Correspondingly, a framework that maps the cloud computing with democratization of resources has been proposed in the upcoming section. Proposed method maps the way cloud computing is leveraged to proliferation of democratic values and empowering its subscribers.

\subsection{Cloud computing democratizing framework}

Cloud computing is offering the services and computing power that has never witnessed before. This paradigm is acting as great leveler and offers the resources without discriminating the subscribers based on caste, creed, religion, location, size of enterprises, etc. In order to relate the aforementioned factors, we have designed the democratizing framework with the help of which we have attempted to prove the way cloud is acting as great leveler and equal opportunity provider.

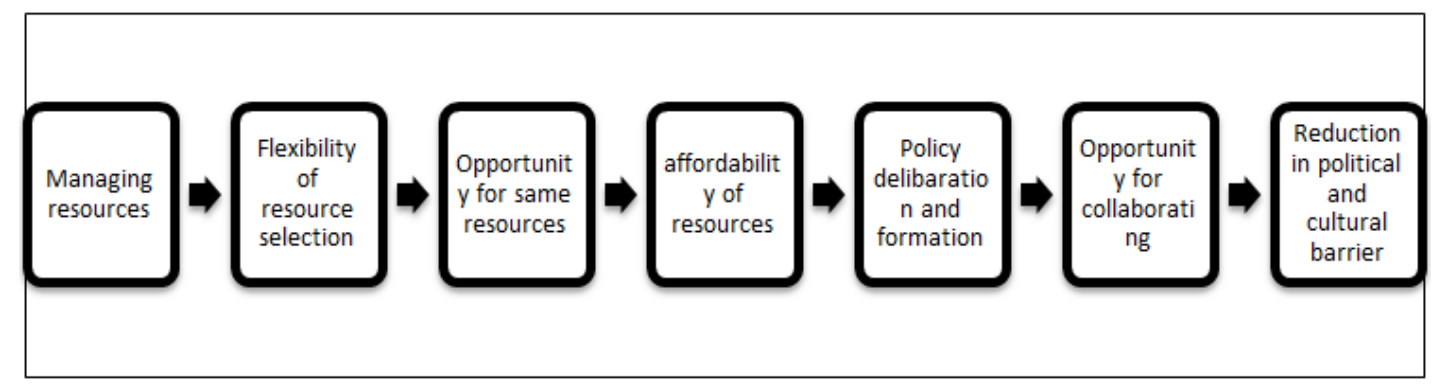

Fig.3. Framework for Democratization of Cloud Resources

Like democratic system that offers freedom to select any kind of job, location for domicile and selection of government; Cloud computing is also democratizing the IT usage in true sense. Upcoming section enumerates the major factors that facilitate in democratizing the IT usage. 


\subsubsection{Managing IT resources}

In today's business scenario, IT is indispensable. However, organizations aiming to embrace IT need huge upfront cost in procuring and managing the following resources:

- Servers

- Selecting the skilled personnel

- Space to keep the resources

- Power requirement

- Physical and software based security

- Expenditure on salary

Consequently, major share of the fund is soaked by the aforementioned requirements. According to the Gartner, traditional IT organizations are incurring 2/3 of their expenditure in routine operations (Wilcox, 2011). As a result, organizations are left with no choice or minimum choice for infrastructure development. Cloud usage would result in 35 to 50 percent saving in operational and infrastructural saving (Wilcox, 2011).

Affordability of large enterprises is more, therefore, huge upfront cost may be manageable for them but for individual users and small enterprises it is nightmare. They are left with either no IT option or make local arrangement that is most of the time is less reliable, low in performance and requires developing a new application for each solution as the organization grows. Consequently, expenditure increases and solutions offered are neither long lasting nor comprehensive. With the emergence of cloud computing, upfront cost is replaced with operating cost. Hence, incorporating IT that is greatly secured, huge resources and additional space for the resources is feasible for the individual users as well as small and medium enterprises.

\subsubsection{Flexibility of resource selection}

Cloud computing is democratic in offering the resources to the subscribers. It offers great flexibility to the users by offering multiple option of resource selection. User can opt for the resources that are most appropriate to them. For instance, in IaaS model, wide varieties of plans are offered that vary based on the Processor's computing power, RAM capacity, bandwidth, etc. Several of these plans offered by the prominent cloud providers include:

A. Microsoft: The IT giant Microsoft is offering variety of plans in order to target the wide variety of users. Microsoft is offering three instances with different level of computing capabilities:

- Small instance (01 core, 1.75 GB RAM)

- Medium instance (02 core, 3.5 GB RAM)

- Large instance( 04 core, 7 GB RAM)

B. Amazon: Amazon has unique position and considered as leader in public cloud domain. Amazon is offering range of services to its subscribers. These services are ranging from IaaS to SaaS. No discrimination is made on the basis of size; revenue of the subscribing enterprises. Therefore, it is profoundly beneficial for SME's and individuals those who have started their own business with limited fund.

Amazon offers huge flexibility in its IaaS service model. Users can opt a plan that is most appropriate for their requirement. Resources are offered with the nomenclature of instances, and categorized based on the size 
as mentioned in the below:

- Small instance

- Medium instance

- Large instance

- Very large instance

Instances with smaller size are less expensive, consequently, affordability of the individual increases significantly. Beyond, subscribers can also realize the advantage of spot instances where the prices are governed as per the need of the market. Option for reserved instances also exist that can be opted by the cloud user. Again no discrimination is made whatsoever. Everyone is free to decide on the type of instance subscribed.

\subsubsection{Opportunity for similar resource usage}

In the legacy system, individual and small enterprises are unable to purchase the software from the mature IT companies since the software developed by the mature companies were expensive. Consequently, to reduce the expenditure such organizations are compelled to purchase from the companies/individuals who worked on adhoc basis. These software were less flexible at the same time could be utilized for the limited purpose only. Due to the utility based model of cloud computing, even the smaller enterprises can subscribe for the same software that can be utilized by the established and reputed enterprises. New startup organizations or the individuals are utilizing the reliable, efficient resources that were possible to the established enterprises. Correspondingly, it can be inferred that opportunity to compete has increased significantly for the SME's and individuals.

\subsubsection{Affordable Resources provisioning}

Cost comparison study has been carried out by the geek (Roggero, 2013). Study revealed that cost of onpremises resources is much higher particularly in the first year. However, expenditure in own maintained resources reduces in second and third year. Considering the case of cloud computing, cost remains stationary for the period upto three year. With the emergence of virtualization, cost of the on premises based resources has reduced drastically; still, it is profoundly higher relative to the cloud based usage.

Beyond the cloud and on-premises based usage, dedicated hosting yields moderate result related to the cost and higher degree of security relative to the cloud computing. Cost comparison for the on-premises model, dedicated hosting and cloud computing has been illustrated in the figure 4(Roggero, 2013).

\subsubsection{Social Media and Collaboration tool/ Policy formulation and deliberation}

In democracy, discussions and deliberations are the key for the formulation of a policy. With the increasing use of social media, people are also initiating the discussion of contemporary issues on the social media. The topics that go viral additional resources are also needed. To serve such requirement, cloud computing is well suited. Social networks can be hosted on cloud that is extremely useful in proliferation of the democratic values of discussion and uniting for a cause. Similarly, forms can be hosted on a cloud in order to obtain the feedback from the citizens and other stakeholders on specific issue. Once many people discuss the same policy, new ideas emerges which can be further incorporated in the upcoming policy in order to ensure the wider participation and acceptability. 


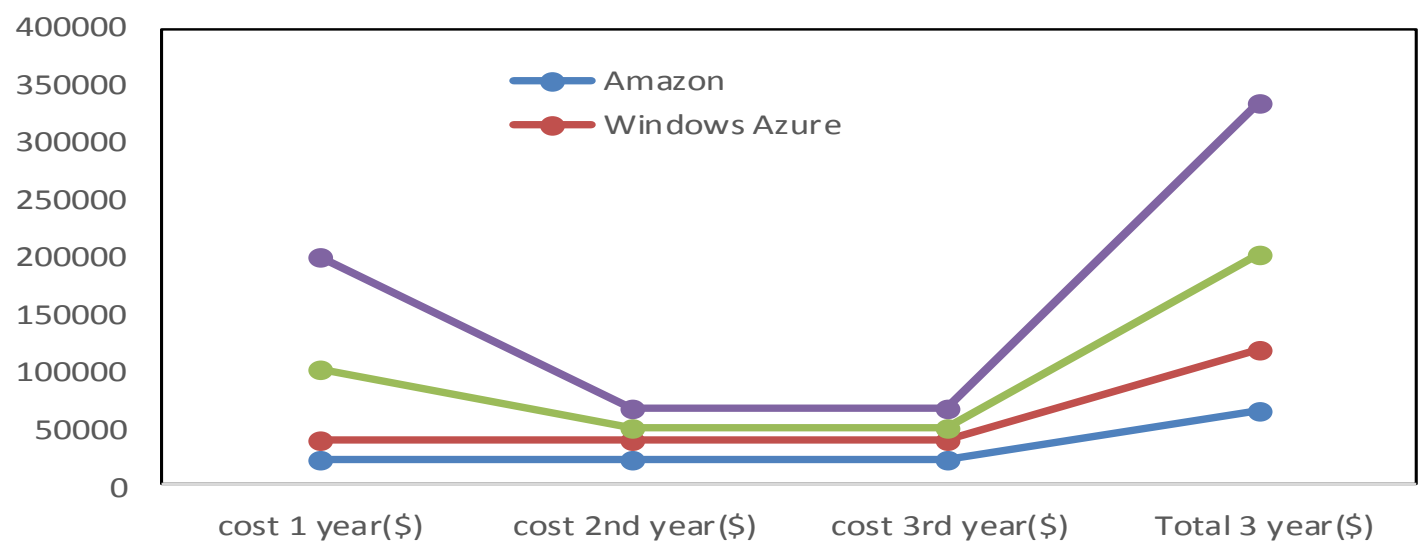

Fig.4. Expenses in Cloud, Dedicated Hosting, and on Premises Model (Wilcox, 2011)

\subsubsection{Democracy in resource sharing}

In a democratic setup, people are empowered to select the government that in turn executes the welfare and development plan on people's behalf. Similarly, cloud computing need to empower the user to select the infrastructure, development environment and applications for their usage and pay on the usage. Infrastructure that was day dream for small and medium enterprises is now readily available on utility based model. Emerging entrepreneurs are able to obtain the much needed IT support from the cloud services.

Cloud computing support multi-tenancy where all the subscribers are utilizing the same cloud resources offered. Here again no discrimination is made on resource usage.

Choice to select the data center is also vested with the subscribers. It can be equally adopted by the enterprises for the discussion and deliberation among the team members. Several social networks are emerging that are hosted on cloud platform, for instance, Oracle social network (Oracle, 2015).. Unnecessary emails can be reduced to a great extent by utilizing the social network. Oracle social network can reduce the $60 \%$ unnecessary emails and 50\% of the time needed in conducting the meeting (Oracle, 2015).

\subsubsection{Cloud computing in employee democracy}

Cloud computing is transforming the employee's working. It is acting as one of the major factors which have transformed the physical offices into virtual office that allows employees to work from home or from anywhere, in addition to the physical office. Consequently, both the employee and employers are equally benefitted. Employers are benefitted by way of saving that result due to less office space that leads to less rental cost; whereas employees are benefitted by saving the commutation expenditure and time.

\subsubsection{Reducing the political and cultural barrier}

Cloud computing is acting as a great leveler and offers the same resources to all the subscribers irrespective of their revenue, region, or the culture. Subscribers opting for the same plan enjoy the same resources. Difference if any is due to the technical limitations. For instance, resources subscribed by the subscriber in the developed country may face minimum latency in content delivery since located near the data center, whereas more time is needed for the subscriber located in Asia due to distance.

Accessibility of the resources across the world without discriminating the region and culture promotes the 
political harmony among the various nations since they are compelled to form the policy in order to protect the interest of all the stakeholders.

\subsubsection{Community cloud}

Major democratic countries have adopted the federal structure to unite their states. In such structure federal laws are customized to the local need without hindering the union law. Similarly, community model of cloud supports the federalism or hierarchical based functioning where additional rules can be included or existing rules can be modified to fulfill the specific/local need irrespective of the balance sheet and geographic location. It enables the organization to share the resources among them as well as outside their organization. All these stake holders can collaborate and exchange information. Sharing and contribution is not restricted to a single country instead contributors from various countries can participate. Therefore, cloud is not restricted to political and cultural barriers.

\section{A. Cloud Initiative by Government}

Recently, governments of many countries have included governance as key agenda. The main objective is to improve the transparency with the help of good governance. Information Technology has to play the key role in the good governance. However, maintaining the IT resources and employing the skilled manpower, and securing the IT resources needed require huge investment. Worst part of the resources owned is that the majority of the time IT resources remain underutilized. Consequently, cloud is emerging as a great alternative in offering the IT resources.

\subsection{Government specific clouds}

To meet the government requirement, government specific cloud has been developed to meet the requirement. Government organizations are adopting the government specific clouds such as Amazon government clouds or they are developing their own cloud to meet their specific needs (Paoli, 2014). Recently, People's expectations have increased from the government many folds. Consequently, government needs to be more open that signifies that information can be accessed at any-time, by anyone and from anywhere using wide variety of devices that include computer, Laptop, Smartphones, tablets, etc.

\subsubsection{Cloud application for governance}

To realize the democratic values, it is equally important that IT facilitate the government and the citizens can interface with the government ICT model. Considering this in mind, numerous countries have adopted cloud or considering adopting cloud computing. Many of the countries are also offering the applications through their AppStore. Users can map their requirement to any of these applications.

Many cloud providers are offering variety of applications that can be utilized for the efficient governance. Government from different countries have initiated their plan to adopt the cloud computing. US, UK, India, etc. are the prominent countries under this category. Major aim for such initiative is to reduce the government expenditure, offering efficient services to the citizen at ease, or providing the information (Zissis \& Lekkas, 2011). Major initiatives of leading countries in the cloud area have been discussed in the upcoming sub-section.

\subsubsection{US Government Cloud}

US government, led by Vivek Kundra has developed the strategy for cloud computing called as "Cloud first mandate". The key objective is to identify the services that can be migrated on cloud in order to reduce the government expenditure from $\$ 80 \mathrm{bn}$ to $\$ 20$ bn by 2015 (Dixon, 2012). The other initiative is pertaining to the 
open cloud. It is believed that open cloud can engage more citizens in order to obtain their opinions, increase participation and collaboration. Open cloud can boost citizens participation in policy formation. Similar drive is initiated by US Govt. in their concept 18F (Paoli, 2014; Open Government Initiative, 2009).

\subsubsection{UK government initiative}

UK Government has initiated the G-cloud that is meant for the Government offices and enables them to utilize cloud computing for the public usage. According to an estimate, government cloud will be extremely useful in the reduction of IT cost. UK Government has also started a cloudstore that is used to purchase the items. According to the G-cloud, UK's 75\% of the suppliers are under the category of SME. Therefore, it offers huge potential growth to the SME's and new debutant in the business. UK government has also started the AppStore to offer various applications.

\subsubsection{Indian Government Initiative}

Recently, Indian government under the aegis of Department of information and communication technology (Diety) of India has launched their cloud services by the name of 'Meghraj' (Hindi name of cloud). The key objective of this project was to collaborate with the other states in order to share the information. In addition, government is also discovering the potential to use this technology in education and healthcare in order to provide the world class education and health services at affordable price at the same time to uplift the living standard of its citizen

\subsubsection{Initiative by the Australian government}

Recently, a study is conducted to provide Sida and other stakeholders the insight of ICT usage for the enhancement of democracy with focus on empowerment. Significance of cloud computing can be estimated by the facts that the upcoming policies that are framed by the various nations to safeguards the interest of all the stakeholders are derived from the information available on internet. For instance, Australian government has formed the policy on cloud computing titling "Australian government cloud computing policy".

\subsubsection{Other initiatives}

This form of cloud is extremely useful in supporting and promoting the federated structure followed in many of the countries including India, UK, USA, etc. by using ICT. Consequently, it can be concluded that the community model is increasingly suitable for the federal based structure in democracy

\section{Conclusions}

Cloud computing has gained huge popularity and adoptability in the recent past. Wide varieties of resources are offered by it at affordable rates. More importantly offered resources are provided to the users without discriminating on their balance sheet, location of demand, time of demand, etc. Considering the responsibility of democratic government towards their citizens, cloud providers are also offering their services dedicated solely for the government. A number of governments have also realized the significance of cloud, correspondingly many of them have initiated to offer their public services using cloud paradigm. Measures adopted will greatly help in proliferation of the democratic values, good governance and offering equal opportunity to everyone. Correspondingly, it can be concluded that cloud computing is a symbol of democracy in technology and will continue to offer growth opportunity to all, particularly to the individuals and SMEs with limited resources. 


\section{Acknowledgements}

Author extends his thanks to all anonymous reviewer(s) whose valuable input has helped us to improve the manuscript before leaving at this stage.

\section{References}

[1] Armbrust, M., Fox, A., Griffith, R., Joseph, A., Katz, R., Konwinski, A., Lee, G., Patterson, D.,Rabkin, A., Stoica, I. \&Zaharia, M.(2009), “ Above the Clouds: A Berkeley View of Cloud Computing”, University of California at Berkley, USA. Technical Report UCB/EECS-2009-28.

[2] Australian government cloud computing policy version 2.0, 2013.

[3] Buyya, R., Yeo, C.S., Venugopal, S., Broberg, J. \& Brandic, I. (2009). Cloud computing and emerging IT platforms: Vision, hype, and reality for delivering computing as the 5th utility. Future Generation Computer Systems, 25 (6) 599-616.

[4] Cloud security alliance (Dec, 2009), "Security Guidance for Critical Areas of Focus in Cloud Computing", V2.1 available at https://cloudsecurityalliance.org/csaguide.pdf

[5] Curtis, J., (Oct, 2014)."10 top cloud computing providers for 2014", available at http://www.cbronline. com/cloud (accessed on 28 Dec 2014).

[6] Dixon, J.S. (2012). Government: cloud first mandate. Available at www.thecloudmagzine.com/government-cloud-mandate).

[7] Islam, S.Keunga, J., Lee, K. \& Liu, A. (2012). Empirical prediction models for adaptive resource provisioning in the cloud. Future Generation Computer Systems, 28, 155-162.

[8] Li,A.,Yang, X.,Kandula, S., Zhang, M.(2010).Cloudcmp: comparing public cloud providers. In proceeding of 10th ACM SIGCOMM conferment on Internet Measurement, New York, NY, USA, pp. 114.

[9] Lin, G., Fu, D., Zhu, J., \& Dasmalchi, G. (2009).Cloud computing: It as a service. IEEE IT Professional, 12(5), 36-41.

[10] Open Government Iniative (2009). Memorandum for the heads of executive departments and agencies, Washington, US.

[11] Paoli, J. (2014, Oct.). "Digital government, openness and open cloud--new cornerstone of democracy", available at http://fcw.com accessed on (11 Jan 2015).

[12] Rappa, M.A. (2004). The utility business model and the future of computing services. IBM Systems Journal, 43 (1), 32-42.

[13] Risch, M. \& Altmann, J. (2009). Enabling Open Cloud Markets Through WS-Agreement Extensions. Service Level Agreements in Grids Workshop, in conjunction with GRID 2009, in: CoreGRID Springer Series, Banff, Canada.

[14] Risch, M., Altmann, J.,Guo, L., Fleming, A.\& Courcoubetis, C. (2009). The GridEcon platform:a business scenario testbed for commercial cloud services, in: 6th International workshop on Grid Economics and Business Models, Delft, The Netherlands, August.

[15] Roggero, H. (2013),"Sample Pricing Comparison: On-Premise vs. Private Hosting vs. Cloud Computing", available at http://geekswithblogs.net/hroggero/archive/2013/02/18/samplepricing-comparison-amazonaws-and-windows-azure.aspx (accessed on 04 Feb 2014).

[16] Singh, J. \& Kumar, V.(2014), "Multi-disciplinary research issues in cloud computing", Journal of information technology and research, Vol. 7, No. 3, pp. 32-53, 2014, DOI:10.4018/jitr.2014070103.

[17] Singh, J. \& Kumar, V.(2011), "Assessment of security risks in merging the private and public cloud", Asia pacific business review, Vol. VII, No. 4, 2011, pp. 180-190. (ISSN: 0973-2470). 
[18] Singh, J. \& Kumar, V.(2013,b) "Business Opportunities and Challenges for Cloud Based ERP Solutions", Asia Pacific Marketing review, Vol.2, Issue 1,2013 pp. 86-95, 2013.

[19] Singh, J. (2014, b), "Cloud computing, for beginner to researcher", CS an Amazon company, USA, ISBN13: $978-1495221682$.

[20] Wilcox, J. (2011). Gartner: Most CIOs have their head in the cloud. Retrieved from http://betanews.com/ 2011/01/24/gartner-most-cios-have-their-heads-in-theclouds/.

[21] Yeo, C.S., Buyya, R., Assuncao, M.D. de, Yu, J.,Sulistio, A.,Venugopal, S. \& Placek, M. Utility computing on global grids. In: H. Bidgoli (Ed.), Handbook of Computer Networks, John Wiley and Sons, Hoboken, NJ, USA, 2007.

[22] Zissis, D. \& Lekkas, D. (2011). Securing e-Government and e-Voting with an open cloud computing architecture. Government Information Quarterly, Vol. 28 pp. 239-251.

\section{Authors' Profiles}

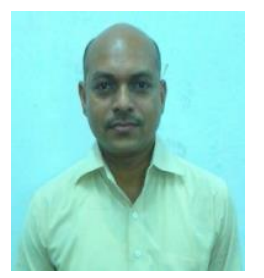

Jitendra Singh has Pursued his PhD (Computer Science) in the area of cloud computing. $\mathrm{He}$ is having over 12 years of experience in the various domains of Information technology. Prior to doctorate, he has pursued the master in computer applications as well as masters in computer technology. In addition, he has also qualified the prestigious UGC-NET examination conducted by 'UGC India' in the year 2006.

As a faculty engaged in teaching to the students of Bachelor's and master's Degree of several premier Universities of India that includes University of Delhi, IP University, IGNOU, etc. as well as foreign University including the Stratford University, USA, etc. to name a few. He is also contributing as a member of several committees which are monitoring and reviewing the curriculum implementation.

He authored more than 20 research articles on cloud computing security, performance, etc. in which several of them are published in the leading research journals and conferences. Besides, he has authored two books pertaining to the computer science, one on the cloud computing titling "cloud computing for beginner and researcher' and the other one on data structure titling "Data structure simplified: Implementation using C ++ ".

How to cite this paper: Jitendra Singh,"Democratizing IT: A Cloud Computing Framework", International Journal of Education and Management Engineering(IJEME), Vol.6, No.2, pp.1-13, 2016.DOI: 10.5815/ijeme.2016.02.01 